\title{
Non-Fermi-Liquid Behavior from the Fermi-Liquid Approach
}

\author{
V. A. Khodel,${ }^{1,2}$ J. W. Clark,${ }^{1}$ H. Li,${ }^{1}$ V. M. Yakovenko, ${ }^{3}$ and M. V. Zverev ${ }^{2}$ \\ ${ }^{1}$ McDonnell Center for the Space Science and Department of Physics, \\ Washington University, St.Louis, MO 63130, USA \\ ${ }^{2}$ Russian Research Center Kurchatov Institute, \\ Moscow, 123182 Russia \\ ${ }^{3}$ Condensed Matter Theory Center and Center for Superconductivity Research, \\ Department of Physics, University of Maryland, \\ College Park, Maryland 20742-4111, USA
}

(Dated: September 15, 2021)

\begin{abstract}
Non-Fermi liquid behavior of strongly correlated Fermi systems is derived within the Landau approach. We attribute this behavior to a phase transition associated with a rearrangement of the Landau state that leads to flattening of a portion of the single-particle spectrum $\epsilon(\mathbf{p})$ in the vicinity of the Fermi surface. We demonstrate that the quasiparticle subsystem responsible for the flat spectrum possesses the same thermodynamic properties as a gas of localized spins. Theoretical results compare favorably with available experimental data. While departing radically from prevalent views on the origin of non-Fermi-liquid behavior, the theory advanced here is nevertheless a conservative one of in continuing to operate within the general framework of Landau theory.

PACS numbers: 71.10.Hf, 71.27.+a, 71.10.Ay
\end{abstract}

\section{INTRODUCTION}

This year is a jubilee for Fermi liquid (FL) theory. Fifty years ago L. D. Landau published an article $\frac{1}{}$ devoted to evaluation of thermodynamic properties of three-dimensional (3D) liquid ${ }^{3} \mathrm{He}$, in which he postulated a one-to-one correspondence between the totality of real, decaying single-particle (sp) excitations of a Fermi liquid and a system of immortal interacting quasiparticles. Two distinguishing features specify this system: (i) its quasiparticle number is equal to the particle number (the so-called Landau-Luttinger theorem), and (ii) its entropy $S$, given by the ideal-Fermi-gas combinatorial expression, coincides with the entropy of the actual helium system. Treating the ground-state energy $E_{0}$ as a functional of the quasiparticle momentum distribution $n(p)$, Landau derived a formula

$$
n(p, T)=\left[1+e^{\epsilon(p) / T}\right]^{-1}
$$

for the quasiparticle momentum distribution $n(p, T)$ that resembles the corresponding Fermi-Dirac formula for the ideal Fermi gas. However, the quasiparticle energy $\epsilon(p)=$ $\delta \Omega / \delta n(p)$ is a variational derivative of the thermodynamic potential $\Omega=E-\mu N$, where $E$ is the groundstate energy and $\mu$, the chemical potential, with respect to the momentum distribution $n(p)$, rather than the bare sp energy $\epsilon_{p}^{0}$ entering the respective Fermi-Dirac formula. As we shall see, in strongly correlated Fermi systems, this noncoincidence is crucial.

Landau's quasiparticle pattern of low-temperature phenomena in Fermi liquids is universally recognized as a cornerstone of condensed-matter theory. However, a qwirk of fate is that although originally FL theory was created for the explanation of properties of 3D liquid ${ }^{3} \mathrm{He}$, discrepancies between theoretical predictions and experimental data on the low- $T$ spin susceptibility $\chi(T)$ and the ratio $C(T) / T$ of the specific heat $C(T)$ to temperature $T$ (both these must be constant in FL theory) first came to right in this liquid. The deviations, rather small at extremely low $T$, increase with temperature. In their book, Nozières and Pines ${ }^{2}$ attribute the departures to the damping of sp excitations. During more than thirty years this claim was regarded as a terminal diagnosis of FL theory. However, the situation was turned upside down about ten years ago when $2 \mathrm{D}$ liquid ${ }^{3} \mathrm{He}$ was studied quantitatively in a region of densities $\rho$ where this system gradually evolves from a weakly correlated gas to a strongly correlated liquid. $\stackrel{3.4}{=}$ The experimental data turned out to be even more challenging to interpret than those for the 3D counterpart. Indeed, in the density region where strong correlations set in, departures from predictions of FL theory exhibit themselves more strongly, the lower the temperature. This definitely rules out damping of sp excitations as the cause for failure of FL theory, since damping vanishes as $T \rightarrow 0$.

The departures in question occur in the density region where the FL effective mass $M^{*}(\rho)$, extracted from the 2D liquid ${ }^{3} \mathrm{He}$ specific heat data ${ }^{4}$ using the FL formula $C_{\mathrm{FL}}(T) / T=p_{F} M^{*} / 3$, is found to be enhanced, with the enhancement rapidly increasing as $\rho$ approaches a critical value $\rho_{s} \simeq 0.072 \AA^{-2}$, beyond which $2 \mathrm{D}$ liquid ${ }^{3} \mathrm{He}$ solidifies. Significantly, the behavior of $\chi(T)$ changes drastically well before $\rho$ reaches $\rho_{s}$ : the product $\chi(T) T$ ceases to vanish at $T \rightarrow 0$, in agreement with the Curie law and in contrast to predictions of standard FL theory. In doing so, the value of this product gradually increases as $\rho \rightarrow \rho_{s}$. Analogous behavior has been observed for other strongly correlated Fermi liquids (see e.g. Ref. $\underline{5}$ ).

Various theories have been suggested in attempts to identify the source of non-Fermi-liquid (NFL) behavior in strongly correlated Fermi systems. Many of them are based on a theory advanced in Refs. 6,7,7, in which the 
NFL behavior is attributed to spin fluctuations. However, the spin-fluctuation scenario fails to explain experimental results for thermodynamic properties of heavyfermion metals in the vicinity of the so-called quantum critical point, especially in the presence of external magnetic fields. $.9,9,10,11$

The gradually emerging Curie behavior of $\chi(T)$ in $2 \mathrm{D}$ liquid ${ }^{3} \mathrm{He}$ is sometimes attributed to a localization phase transition. Unfortunately for this explanation, a homogeneous system without impurities has no more chance to be slightly localized than a women has to be slightly pregnant. The Curie-Weiss behavior of $\chi(T)$ in heavy-fermion metals is ordinarily described within the Anderson $s-d$ model, in which localized $d$ states acquire a nonzero spin as a result of interaction with delocalized $s$ electrons. However, within this scenario it is hard to reconcile the localization of electrons with Cooper pairing, which is found to be unexpectedly strong in some cases $\underline{12}$

We shall argue that the occurrence of the Curie-like term in low-temperature behavior of the magnetic susceptibility $\chi(T)$ of a homogeneous Fermi liquid, which gradually evolves under change of input parameters, is a signature of a phase transition known by fermion condensation. This phase transition is associated with a rearrangement of single-particle degrees of freedom, rather than collective ones.

\section{REARRANGEMENT OF SP DEGREES OF FREEDOM IN FINITE FERMI SYSTEMS}

It is instructive to begin studying such a rearrangement in finite Fermi systems, in which damping of singleparticle excitations does not occur. This closes off one favorite escape route of critical readers and referees. It is the conventional wisdom of textbooks that under variation of input parameters, two sp levels may repel or cross one other. However, as we shall demonstrate, the familiar dichotomy misses a further alternative: levels can in fact merge $\stackrel{13}{\underline{13}}$ This phenomenon is made possible by the variation of sp energies with level occupation numbers - a property central to Landau theory. A primary condition for merging to occur is that the Landau-Migdal interaction function $f$ is repulsive in coordinate space,$\frac{13}{n}$ which holds for the effective $n n$ and $p p$ interactions in the nuclear interior ${ }^{14}$ and for the electron-electron interaction in atoms.

Consider a schematic model involving three equidistant neutron levels, separated by an energy distance $D$ in an open shell of a spherical nucleus. The levels are denoted,- 0 , and + , in order of increasing energy. The sp energies $\epsilon_{\lambda}$ and wave functions $\varphi_{\lambda}(\mathbf{r})=R_{n l}(r) \Phi_{j l m}(\mathbf{n})$ are solutions of

$$
\left[p^{2} / 2 M+\Sigma(\mathbf{r}, \mathbf{p})\right] \varphi_{\lambda}(\mathbf{r})=\epsilon_{\lambda} \varphi_{\lambda}(\mathbf{r}),
$$

where $\Sigma$ stands for the self-energy. In even-even spherical nuclei, which in their ground states have total angular momentum $J=0$ due to pairing correlations, the energies $\epsilon_{\lambda}$ are independent of the magnetic quantum number $m$ associated with the total sp angular momentum $j$. We suppose that the level - is filled, the level + is empty, and $N$ neutrons are added to the level 0 , changing the density $\rho(r)$ by $\delta \rho(r)=N R_{n_{0} l_{0}}^{2}(r) / 4 \pi$.

In what follows, we shall retain only a major, spinand momentum-independent part $V$ of the self-energy $\Sigma$ and a primary, $\delta(r)$-like portion of the Landau-Migdal interaction function $f$. Accordingly, the FL relation between $\Sigma$ and $\rho$ responsible for the variation of $\epsilon_{\lambda}(n)$ with $n$ reduces to $\underline{14}$

$$
\delta V(r)=f[\rho(r)] \delta \rho(r) .
$$

When particles are added to the system, all energy levels are shifted somewhat, but the level that receives the particles is affected more strongly than the others. For the sake of simplicity, the diagonal and nondiagonal matrix elements of $f$ are assigned the respective values

$$
\begin{gathered}
u=\int R_{n l}^{2}(r) f[\rho(r)] R_{n l}^{2}(r) r^{2} d r / 4 \pi, \\
w=\int R_{n l}^{2}(r) f[\rho(r)] R_{n_{1} l_{1}}^{2}(r) r^{2} d r / 4 \pi,
\end{gathered}
$$

independently of the quantum numbers $n l, n_{1} l_{1}$.

Based on these assumptions and results, the dimensionless shifts $\xi_{k}(N)=\left[\epsilon_{k}(N)-\epsilon_{k}(0)\right] / D$ for $k=0,+,-$ are given by

$$
\xi_{0}(N)=n_{0} U, \quad \xi_{+}(N)=\xi_{-}(N)=n_{0} W,
$$

where $n_{k}=N_{k} /\left(2 j_{k}+1\right)$ is the occupation number of the level $k, U=u\left(2 j_{0}+1\right) / D$, and $W=w\left(2 j_{0}+1\right) / D$. It is readily verified that if $f p_{F} M / \pi^{2} \sim 1$, where $p_{F}=$ $\sqrt{2 M \epsilon_{F}}$ and $\epsilon_{F}$ is the Fermi energy, then the first of the integrals (4) has a value $u \simeq \epsilon_{F} / A$ and therefore $U \sim 1$, since $D \sim \epsilon_{F} / A^{2 / 3}$ in spherical nuclei.

According to Eqs. (5) at $(U-W)>1$, the difference $d(N)=1+\xi_{+}(N)-\xi_{0}(N)$ changes sign at $n_{0 c}=1 /(U-W)$, before filling of the level + is complete. At $n_{0}>n_{0 c}$, in the standard scenario provided by Hartree-Fock theory, all added quasiparticles must resettle into the empty sp level + . However, not all of the quasiparticles can take part in the migration process, since the situation would then be reversed, and the roles of the levels interchanged: the formerly empty level, lying above the formerly occupied one, would have the maximum positive energy shift, rendering migration impossible. Thus, the standard Fermi-liquid filling scenario, which prescribes that one and only one sp level lying exactly at the Fermi surface can remain unfilled, while all others must be completely occupied or empty, encounters a catastrophe.

This catastrophe is resolved as follows 13.15 . Migration occurs until the sp energies of the two levels in play coincide. As a result, both of the levels, 0 and + , become 


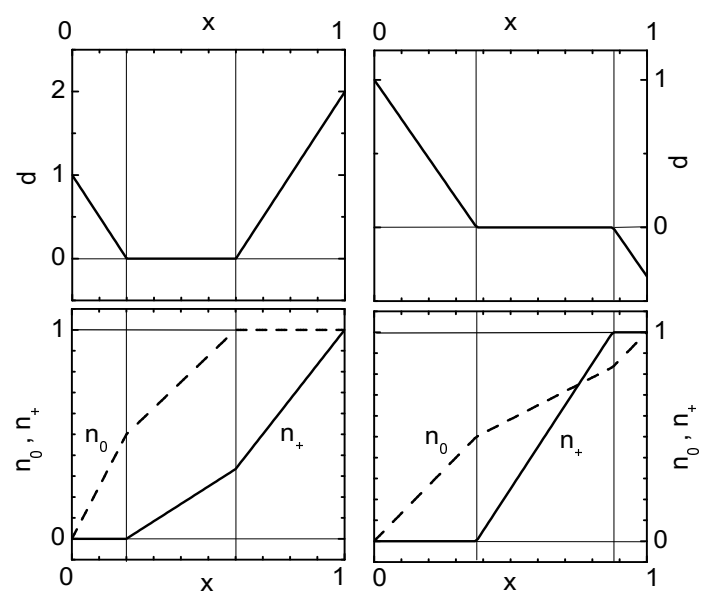

FIG. 1: Top panels: Dimensionless distance $d=\left(\epsilon_{+}-\epsilon_{0}\right) / D$ between levels + and 0 as a function of the ratio $x=N /\left(2 j_{0}+\right.$ $\left.2 j_{+}+2\right)$. Lower panels: Occupation numbers $n_{k}$ for levels 0 and + . Input parameters: $U=V=3, W=1$. For the left column, the ratio $r \equiv\left(2 j_{0}+1\right) /\left(2 j_{+}+1\right)=2 / 3$; for the right, $r=3$.

partially occupied - an impossible situation for the standard Landau state. Solution of the problem reduces to finding the minimum of the relevant energy functional

$E_{0}=\epsilon_{0}(0) N_{0}+\epsilon_{+}(0) N_{+}+\frac{1}{2}\left[u\left(N_{0}^{2}+N_{+}^{2}\right)+2 w N_{0} N_{+}\right]$

with $N_{k}=\sum_{m} n_{k m}$, through a variational condition

$$
\frac{\delta E_{0}}{\delta n_{0 m}}=\frac{\delta E}{\delta n_{+m_{1}}}=\mu, \quad \forall m, m_{1},
$$

where $\mu$ is the chemical potential. Such a condition first appeared in Ref. 15, where homogeneous Fermi systems were addressed without attention to the degeneracy of $\mathrm{sp}$ levels. Eqs. (7) are conveniently rewritten as conditions

$$
\begin{aligned}
& \epsilon_{0}(N)=\epsilon_{0}(0)+N_{0} u+N_{+} w=\mu, \\
& \epsilon_{+}(N)=\epsilon_{+}(0)+N_{0} w+N_{+} u=\mu
\end{aligned}
$$

for coincidence of the sp energies $\epsilon_{0}$ and $\epsilon_{+}$, which, at $N>N_{c}=\left(2 j_{0}+1\right) /(U-W)$, yield $N_{0}=\frac{1}{2}\left(N+N_{c}\right)$ and $N_{+}=\frac{1}{2}\left(N-N_{c}\right)$.

Results from numerical calculations are plotted in Fig. 1, which consists of two columns, each made up of two plots. The upper panels show the dimensionless ratio $d(x)=\left[\epsilon_{+}(x)-\epsilon_{0}(x)\right] / D$ versus $x=N /\left(2 j_{0}+2 j_{+}+2\right) \epsilon$ $[0,1]$. The lower panels give the occupation numbers $n_{+}(x)$ and $n_{0}(x)$. We observe that there are three different regimes: in two of them $d \neq 0$ and there exist welldefined sp excitations, and in the third, the energies of the levels 0 and + coincide at zero. Passage through the three regimes can be regarded as a second-order phase transition, with the occupation number $n_{+}$treated as an order parameter.
The sp levels remain merged until one of them is completely filled. If the level 0 fills first, as in the left column of Fig. 1, then under further increase of $N$, quasiparticles fill the level + , signaling that the distance $d(N)$ again becomes positive. This behavior resembles the repulsion of two levels of the same symmetry in quantum mechanics, although here one deals with the sp levels of different symmetry. In a case where level + becomes fully occupied before the level 0 does, as in the right column, the distance $d(N)$ becomes negative, and the two levels just cross each other at this point.

In systems without pairing correlations, for example in atoms, a pair of particles added to any sp level with $l \neq 0$ always has total angular momentum $J \neq 0$ (Hund's rule), in principle destroying spherical symmetry and lifting the $m$-degeneracy of the sp energies $\epsilon_{k m}$, thereby complicating the analysis of merging. Here we only sketch the final results (for details, see Ref. 13). The energy functional

$$
E=\sum \epsilon_{k}(0) n_{k m}+\frac{1}{2} \sum f_{k m, k_{1} m_{1}} n_{k m} n_{k_{1} m_{1}}
$$

replaces the functional (6), and the interaction matrix $f_{k m, k_{1} m_{1}}$ replaces the matrix elements (4). The variational equations

$$
\mu=\epsilon_{k}(0)+\sum f_{k m, k_{1} m_{1}} n_{k_{1} m_{1}},
$$

in which the sum runs over some states of the last unfilled shell that undergo merging, are to be solved numerically. Merging provides a qualitative explanation of the fact that the chemical properties of rare-earth elements differ little, in spite of marked variation in atomic numbers. Such an explanation is to some extent complementary to the textbook argument that the collapse of the electron $4 f$-orbital is responsible for the remarkable similarity of the chemical properties of the rare-earth elements.

It is worth noting that if the number of equations (10) to be solved becomes large, reasonable results can be obtained with the replacement of summation by integration to yield $\frac{15}{}$

$$
\mu=\epsilon_{k}^{0}+2 \int f\left(\mathbf{k}, \mathbf{k}_{1}\right) n\left(\mathbf{k}_{1}\right) d^{3} k_{1} /(2 \pi)^{3} .
$$

As will now be demonstrated, the analysis of merging of sp levels in finite systems helps us understand what is going on in infinite matter. Let us consider a model of a heavy-fermion metal in which the sp spectrum, evaluated usually in local-density approximation, is exhausted by (i) a wide band that disperses through the Fermi surface, and (ii) a narrow band situated below the Fermi surface at a distance $D_{n}$. To facilitate the analysis, we assume that only the diagonal matrix element $f_{n n}$ of the interaction function $f$ referring to the narrow band is significant, the others being negligible. The shift $\delta \epsilon_{n}$ in the location of the narrow band due to switching on the intraband interactions is given by a formula $\delta \epsilon_{n}=f_{n n} \rho_{n}$ analogous to Eq. (5), where $\rho_{n}$ is the density of the narrow band. Suppose now that the correction $\delta \epsilon_{n}$ exceeds 
the distance $D_{n}$. In this case, the HF scenario calls for the narrow band to be completely emptied; but then the shift $\delta \epsilon_{n}$ must vanish. To eliminate this inconsistency, it must be the case that only a fraction of the particles leave the narrow band, in just the right proportion to equalize the chemical potentials of the two bands. The feedback mechanism we have described positions the narrow band exactly at the Fermi surface, resolving a long-standing problem with the LDA scheme.

\section{QUANTUM CRITICAL POINT IN A HOMOGENEOUS FERMI LIQUID}

In this section, we investigate properties of Fermi liquids in the vicinity of the FL quantum critical point (QCP), i.e. in a region close to the critical density $\rho_{\infty}$ where the effective mass $M^{*}$ diverges. Standard FL theory, which knows nothing about the QCP, tells us that properties of Fermi liquids are similar to those of an ideal Fermi gas, with differences merely involving a numerical factor, the ratio $M^{*} / M$. However, at the critical density $\rho_{\infty}$ where $M^{*}$ diverges, the FL spectrum $\epsilon_{\mathrm{FL}}(p ; \rho)$ vanishes identically, signalling that the standard FL theory requires a cure. Remarkably, the Landau approach itself contains a medicine to treat the disease. The cure lies in the relation 1

$$
\frac{\partial \epsilon(p)}{\partial \mathbf{p}}=\frac{\mathbf{p}}{M}+2 \int f\left(\mathbf{p}, \mathbf{p}_{1}\right) \frac{\partial n\left(\epsilon\left(p_{1}\right)\right)}{\partial \mathbf{p}_{1}} d^{3} p_{1} /(2 \pi)^{3},
$$

which connects the quasiparticle spectrum $\epsilon(p)$ and the momentum distribution $n(\epsilon)$ through the Landau interaction function $f\left(\mathbf{p}, \mathbf{p}_{1}\right)$. Referring back to the preceding section in which we considered the merging of sp levels in finite Fermi systems, this relation has its conceptual counterpart in Eq. (3). From Eq. (12) one finds

$$
M / M^{*}(\rho, T=0)=1-F_{1}^{0}(\rho) / 3,
$$

having introduced the dimensionless first harmonic $F_{1}^{0}(\rho)=f_{1}\left(p_{F}, p_{F}\right) N_{0}$, where $N_{0}=p_{F} M / \pi^{2}$ and $f_{1}\left(p_{F}, p_{F}\right)$ is the first harmonic of $f\left(\mathbf{p}_{1}, \mathbf{p}_{2}\right)$. From Eq. (13) we infer that realization of the divergence of $M^{*}$ hinges on the presence of sufficiently large velocity-dependent components in $f$, since one must have $F_{1}^{0}\left(\rho_{\infty}\right)=3$.

Quantitative studies of the spectrum $\epsilon(p)$ first appeared in connection with the problem of fermion condensation (see below) some forty years after the creation of FL theory. Close to the critical density where $M^{*}(\rho)$ diverges, the group velocity $d \epsilon\left(p ; \rho \rightarrow \rho_{\infty}\right) / d p$ ceases to be constant, acquiring a parabola-like shape. ${ }^{16,17}$ This is seen from Fig. 2, where the microscopically evaluated ${ }^{17}$ sp spectrum and group velocity of a $2 \mathrm{D}$ electron gas are shown.

The structure of the sp spectrum $\epsilon(p, \rho)$ in the vicinity of the Fermi-liquid QCP can be elucidated without resorting to the help of a computer. Instead one expands

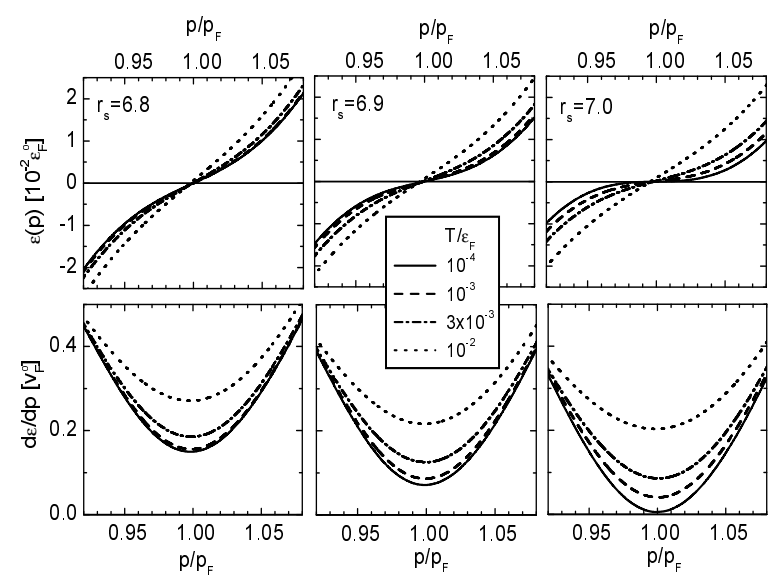

FIG. 2: Single-particle spectrum $\epsilon(p)$ in units of $10^{-2} \epsilon_{F}^{0}$ (top panels) and its derivative $d \epsilon(p) / d p$ in units of $v_{F}^{0}=p_{F} / M$ (bottom panels) for the 2D electron gas at $r_{s}=6.8$ (left column), $r_{s}=6.9$ (middle column), and $r_{s}=7.0$ (right column). Spectra and derivatives are shown as functions of $p / p_{F}$ at four values of temperature in units of $\epsilon_{F}^{0}=p_{F}^{2} / 2 M$.

the relevant quantities on both sides of Eq. (12) in Taylor series, thereby obtaining

$$
d \epsilon / d p \simeq p_{F} / M^{*}(\rho, T)+v_{2}\left(p-p_{F}\right)^{2} / M p_{F} .
$$

Inserting this expression into Eq. (12) and performing rather lengthy algebra, one finds 18

$$
M / M^{*}\left(T, \rho_{\infty}\right) \propto T^{2 / 3} .
$$

Thus, within the Landau approach, the density of states diverges as $T^{-2 / 3}$ when the density $\rho$ approaches $\rho_{\infty}$. The corresponding asymptotic behaviors of the spin susceptibility and the ratio $C(T) / T$ are $\chi\left(T, \rho_{\infty}\right) \propto$ $C(T, \rho) / T \propto T^{-2 / 3}$, while the entropy $S\left(T, \rho_{\infty}\right)$ and the thermal expansion $\beta\left(T, \rho_{\infty}\right)$ behave as $T^{1 / 3}$.

Imposition of a static external magnetic field $H$ brings into play a new dimensionless parameter $R=\mu_{B} H / T$ and opens another arena for testing the Landau approach. The function $n(\epsilon(p))$ entering Eq. (12) is then replaced by $\left[n\left(\epsilon_{+}(p)\right)+n\left(\epsilon_{-}(p)\right] / 2\right.$, where $n\left(\epsilon_{ \pm}(p)\right)=$ $[1+\exp (\epsilon(p) / T \pm R / 2))]^{-1}$. Proceeding as before, one uncovers a scaling behavior

$$
M / M^{*}\left(T, H, \rho_{\infty}\right) \propto T^{2 / 3} a(R)
$$

of the effective mass, where $a(R)$ has been evaluated in a closed form in Ref. 18. In the limit $T \rightarrow 0$ or equivalently $R \rightarrow \infty$, this behavior simplifies, yielding the analytic form ${ }^{18,19} M^{*}\left(T=0, H, \rho_{\infty}\right) \propto H^{2 / 3}$ for the effective mass. Thus, on the metallic side of the phase transition associated with the divergence of the effective mass, imposition of a static magnetic field satisfying $\mu_{B} H>T$ renders the effective mass $M^{*}\left(T, H, \rho_{\infty}\right)$ finite, promoting the recovery of Landau FL theory. This behavior, consistent with the experiment, remains elusive in any 

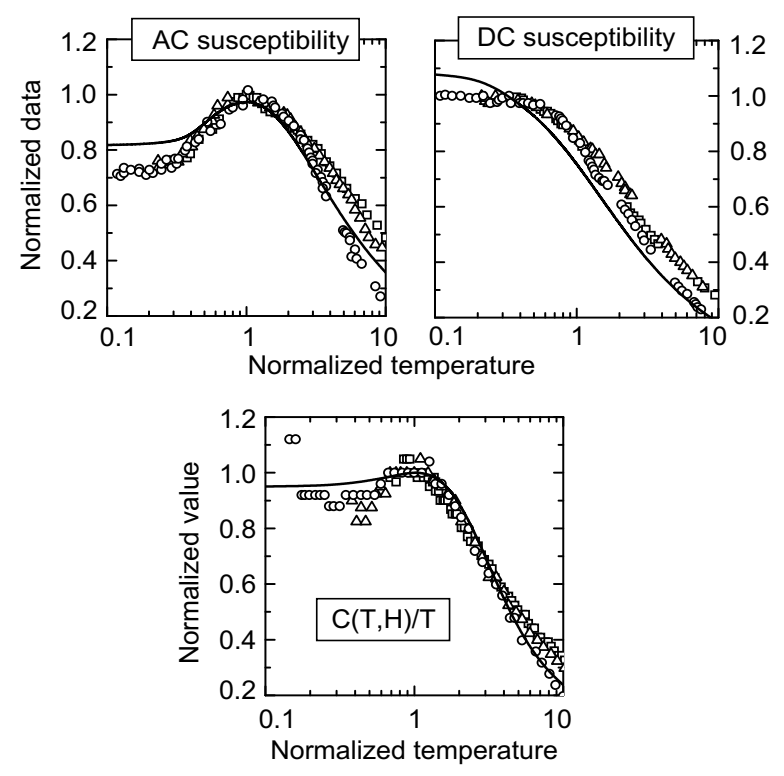

FIG. 3: op panels: Normalized magnetic susceptibility $\chi(T, H) / \chi\left(T_{P}\right)$ (top-left panel) and normalized magnetization $\mathcal{M}(T, H) / \mathcal{M}\left(T_{P}\right)$ (top-right panel) for $\mathrm{CeRu}_{2} \mathrm{Si}_{2}$ in magnetic fields $0.20 \mathrm{mT}$ (squares), $0.39 \mathrm{mT}$ (triangles), and 0.94 mT (circles), plotted against normalized temperature $T / T_{P}$ (Ref. 11), where $T_{P}$ is the temperature at peak susceptibility. The solid curves trace the universal behavior predicted by the present theory. Bottom panel: The normalized ratio $C(T, H) T_{M} / C\left(T_{M}\right) T$ for $\mathrm{YbRh}\left(\mathrm{Si}_{0.95} \mathrm{Ge}_{0.05}\right)_{2}$ in magnetic fields $0.05 \mathrm{~T}$ (squares), $0.1 \mathrm{~T}$ (triangles), and $0.2 \mathrm{~T}$ (circles), versus the normalized temperature $T / T_{M}$ (Ref. 20), where $T_{M}$ is the temperature at maximum ratio $C(T, H) / T$. The solid curve shows the prediction of our theory.

approach involving spin fluctuations as a basic ingredient.

Along the same lines, one finds that close to the QCP, the magnetic moment and AC spin susceptibility display a scaling behavior. Following Ref. 11, Fig. 3 presents the results of numerical calculations of these quantities as functions of the normalized temperatures $T / T_{P}$ and $T / T_{M}$. We see that the model developed here reproduces the experimental scaling behaviors of both the spin susceptibility $\underline{11}$ of the heavy-fermion metal $\mathrm{CeRu}_{2} \mathrm{Si}_{2}$ and the specific heat ${ }^{20}$ of the heavy-fermion compound $\mathrm{YbRh}\left(\mathrm{Si}_{0.95} \mathrm{Ge}_{0.05}\right)_{2}$, without any adjustable parameters.

\section{GOING BEYOND THE POINT OF FERMION CONDENSATION}

For decades, there has been virtually universal acceptance of Landau's hypothesis that in homogeneous systems of fermions there exists a one-to-one $T$-independent correspondence between the sp spectrum $\epsilon(p)$ and the momentum $p$. This postulate (see the text accompanying formula (4) in Ref. 1), implying that the quasiparticle group velocity $d \epsilon / d p$ at the Fermi surface is practically independent of $T$, has been regarded as a cornerstone of FL theory. However, numerical investigations of Eq. (12) performed in recent years $\frac{16}{}$ have demonstrated that this hypothesis is incorrect in the vicinity of the QCP. This flaw was in fact uncovered already in 1990's in connection with an analysis of the necessary condition for stability of the ground state with respect to the rearrangement of the ground-state momentum distribution $n(p)$. The pertinent stability condition reads

$$
\delta E_{0}(n)=2 \int \epsilon(p) \delta n(p) d^{3} p /(2 \pi)^{3} \geq 0,
$$

thus requiring nonnegativity of the variation of the ground-state energy $E_{0}$ under any admissible variation of $n(p)$. In the case of the Landau state where $n(p)$ is simply the Fermi step $n_{F}(p)$, this implies that the sign of $\epsilon(p)$ must coincide with the sign of the difference $p-p_{F}$. However, it turns out that there exists a class of NFL solutions having a completely flat portion $\epsilon(p)=0$ in a region embracing the Fermi surface, $\frac{15,21,22,23}{\underline{2}}$ which in principle provides a different minimum of the functional $E_{0}(n)$. Flattening of the sp spectrum in this way is inescapably accompanied by another peculiarity: occupation numbers $n_{*}(p)$ in the flat region differ from 0 and 1. As a result, the Fermi surface swells from a surface to a volume in $3 \mathrm{D}$ and from a line to a surface in $2 \mathrm{D}$. The phenomenon of swelling of the Fermi surface, first documented in Ref. 15 and called fermion condensation, is its distinctive signature. We have already encountered swelling of the Fermi surface in Sec. 2, where the merging of sp levels was studied. The set of states with $\epsilon(\mathbf{p})=0$ is called the fermion condensate (FC) in transparent analogy with the boson condensate that forms at $T=0$ in a Bose liquid, and in which macroscopically many particles have energy coincident with the chemical potential $\mu$.

The existence of ground states with occupation numbers $0<n_{*}(p)<1$ follows directly from the fundamental idea of the FL approach that $E_{0}$ is a functional of $n(p)$. To see this more clearly, it is instructive to invoke a mathematical correspondence with the functional $E_{0}(\rho)$ of statistical physics. If the interaction is small, the latter attains its minimum for solutions describing gases. However, if the interaction between the particles is sufficiently strong, then nontrivial solutions of the variational condition $\delta \Omega / \delta \rho=0$ on the thermodynamic potential $\Omega=E_{0}-\mu N$ describe liquids. From a mathematical point of view, the functional $\Omega(n)$ must have two types of solutions. Assuredly, in weakly interacting systems, its minimum is attained at $n_{F}(p)=\theta\left(p-p_{F}\right)$, which minimizes the kinetic energy alone. On the other hand, if the potential energy becomes strong, there arise nontrivial solutions of the variational condition

$$
\frac{\delta \Omega}{\delta n(\mathbf{p})}=0, \quad \mathbf{p} \in C,
$$

over a finite domain $C$ adjacent to the Fermi surface $\frac{15,21,22,23}{}$ In Landau's formulation of Fermi-liquid theory, at $T=0$ the derivative $\delta E / \delta n(\mathbf{p})$ is just the sp 
energy, while $\delta \Omega / \delta n(\mathbf{p})$ is the sp energy $\epsilon(\mathbf{p})$ measured from the chemical potential $\mu$. Thus we arrive at the relation

$$
\epsilon(\mathbf{p})=0, \quad \mathbf{p} \in C,
$$

characterizing the fermion condensate.

In contrast to other second-order phase transitions, no symmetry is violated in the FC phase transition, and therefore the choice of the associated order parameter is not immediate. The FC density $n_{*}(p)$ can be treated as such an order parameter. Upon rewriting Eq. (10) as $\epsilon(\mathbf{p}, T)=T \ln [(1-n(\mathbf{p})) / n(\mathbf{p})]$ and inserting into this expression of the FC solution $n_{*}(\mathbf{p})$, one finds ${ }^{22}$

$$
\epsilon(\mathbf{p}, T \rightarrow 0)=T \ln \frac{1-n_{*}(\mathbf{p})}{n_{*}(\mathbf{p})}, \quad \mathbf{p} \in C .
$$

Thus at low $T$, the energy degeneracy of $\mathrm{FC}$ is lifted, the FC plateau in $\epsilon(\mathbf{p})$ being inclined with a slope proportional to temperature. ${ }^{22}$

This salient feature of the FC spectrum exhibits itself in the magnetic susceptibility $\chi(T)$, providing CurieWeiss behavior $\chi(T) \propto 1 / T$ in normal states of systems with a FC. Indeed, upon inserting the function $n_{*}(\mathbf{p})$ into the standard FL relation

$$
\begin{gathered}
\chi_{0}=-2 \mu_{e}^{2} \int \frac{d n\left(\epsilon_{\mathbf{p}}\right)}{d \epsilon_{\mathbf{p}}} d^{3} p /(2 \pi)^{3} \\
\equiv \frac{2 \mu_{e}^{2}}{T} \int n(\mathbf{p})[1-n(\mathbf{p})] d^{3} p /(2 \pi)^{3},
\end{gathered}
$$

we find a Curie contribution to the spin susceptibility 24 given by

$$
\chi_{*}(T)=\frac{\kappa \mu_{e}^{2}}{T}, \quad \kappa=2 \int n_{*}(\mathbf{p})\left[1-n_{*}(\mathbf{p})\right] d^{3} p /(2 \pi)^{3} .
$$

The effective Curie constant in Eq. (22) is reduced by the dimensionless parameter $\kappa$ relative to the standard Curie law $\chi_{0}=\mu_{e}^{2} / T$. Accounting for the spin interaction amplitude $g_{0}$ generates the Curie-Weiss law $\chi(T)=$ $\mu_{e}^{2} \kappa /\left(T-\Theta_{W}\right)$ with a Weiss temperature $\Theta_{W}=g_{0} \kappa \mu_{e}^{2}$. Such a behavior of $\chi(T)$ is observed in ${ }^{3} \mathrm{He}$ films, where the low- $T$ Curie constant is about 4 times smaller than that for high $T$, as shown in Fig. 1 of Ref. 3 , which gives $\kappa \approx 0.25$ in this case.

Another important feature inherent in systems having a FC stems from the fact that the variational condition (18) holds at finite temperatures, $\stackrel{22}{=}$ provided the thermodynamic potential is redefined according to the conventional relation $\Omega=E-\mu N-T S$, where the entropy $S$ is given by the Landau formula

$$
\begin{gathered}
S=-2 \int[n(\mathbf{p}) \ln n(\mathbf{p})+(1-n(\mathbf{p})) \ln (1-n(\mathbf{p}))] \\
\times d^{3} p /(2 \pi)^{3} .
\end{gathered}
$$

Upon inserting here the distribution $n_{*}(p)$, we infer that systems with a FC possess a finite, independent of $T$ entropy $S_{*} \cdot \frac{15,22,23}{1}$ The entropy-excess value, proportional to the total FC density, changes gradually under variation of input parameters. Although it does not contribute to the specific heat, it produces an enormous enhancement of the thermal expansion coefficient $\beta=\partial V / \partial T \equiv-\partial S / \partial P$ and the Grüneisen ratio $\Gamma=$ $\beta / C^{25}$. Experiment ${ }^{26}$ shows that in normal states of several heavy-fermion metals, $\beta$ is in fact temperatureindependent at low $T$ and exceeds typical values for ordinary metals by a factor $10^{3}-10^{4}$. With $\beta \rightarrow$ const and $C(T) \rightarrow 0$, the Grüneisen ratio $\Gamma=\beta / C$ diverges at low $T$, as is observed experimentally $\underset{27}{ }$.

It should be emphasized that the existence of the residual entropy $S_{*}$ at zero temperature contradicts the third law of thermodynamics (the Nernst theorem). To ensure that $S=0$ at $T=0$, localized spins are known to order magnetically due to spin-spin interactions. Similarly, a system with a FC must experience some sort of low-temperature phase transition eliminating the excess entropy $S_{*}$, e.g. the second-order phase transition to a superconducting state $\frac{15}{}$. The presence of the FC in the ground state exhibits itself in a jump $\Delta C \approx 4.7 \kappa$ of the specific heat at $T_{c}$, governed by the $\mathrm{FC}$ parameter $\kappa$ in the Curie law 28 (22). Thus, the ratio $\Delta C / C_{n}$ can be very high when $T_{c}$ is low, because $C_{n} \rightarrow 0$ as $T \rightarrow 0$ while $\Delta C$ remains finite. Such a situation is encountered for example in the heavy-fermion metal $\mathrm{CeCoIn}_{5}$, with $T_{c}=2.3 \mathrm{~K}$ and $\Delta C / C_{n} \approx 4.5$, over three times higher than the BCS value $\stackrel{29}{\underline{2}}$

\section{DISCUSSION}

In any conventional homogeneous Fermi liquid, e.g. liquid ${ }^{3} \mathrm{He}$, the momentum $\mathbf{p}$ of an added particle can be associated with a certain quasiparticle. Similarly, in most spherical odd nuclei, the total angular momentum $J$ in the ground state is carried by an odd quasiparticle. In atomic physics, the electronic configuration of ions of elements belonging to the principal groups of the periodic table repeats that of preceding atoms. By contrast, beyond the point of fermion condensation in homogeneous matter or the point of merging of sp levels in finite systems, the ground state features a multitude of quasiparticle terms and therefore exhibits a different, more complicated character, as in the comparison of a chorus with a dominant soloist.

Notwithstanding evident commonalities, there is a considerable difference between conditions for the "levelmergence" phenomenon in homogeneous Fermi liquids and in finite Fermi systems with degenerate sp levels. In the former, the presence of a significant velocitydependent component in the interaction function $f$ is needed to promote fermion condensation, while in the latter, sp levels can merge even if $f$ is momentumindependent. The reason for this difference is simple: 


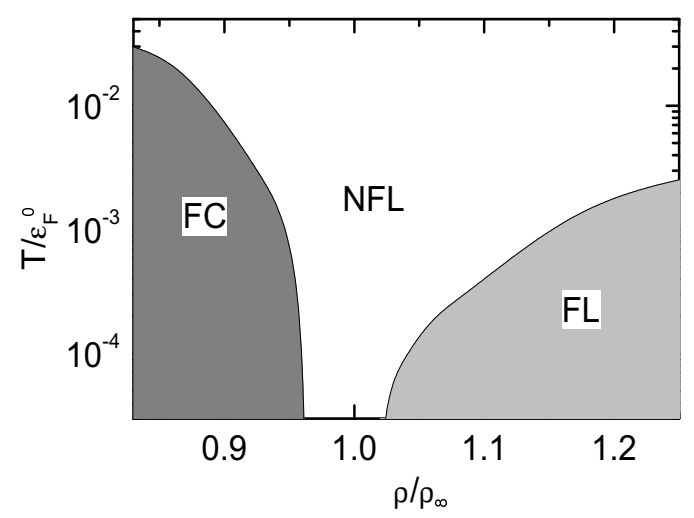

FIG. 4: Phase diagram of $2 \mathrm{D}$ electron gas in $\left(T, \rho / \rho_{\infty}\right)$ variables. Curves show crossovers between usual Fermi liquid (FL), fermion condensate (FC) and non-Fermi liquid (NFL) phase with the critical index $\alpha \simeq 0.6$.

in the homogeneous case, the matrix elements $u$ and $w$ of Eq. (4) are equal to each other, implying zero energy gain due to the rearrangement when velocity-dependent forces are absent.

The salient feature of the fermion condensation phenomenon discussed in this paper is the flattening of the quasiparticle dispersion $\epsilon(\mathbf{p})$ at the Fermi level on the ordered side of the driving phase transition. Prime consequencies of this flattening are: (i) the magnetic susceptibility follows the Curie-Weiss law with an effective Curie constant proportional to the FC parameter $\kappa$, (ii) the entropy has a temperature-independent term $S_{*}$ that greatly increases the thermal expansion coefficient $\beta=-\partial S / \partial P$ at low $T$, (iii) the excess entropy $S_{*}$ released below the superconducting transition temperature $T_{c}$ dramatically reduces $\beta$, enhancing the specificheat jump $\Delta C / C_{n}$. All these features go unexplained in any other microscopic approach.

On the disordered side of the transition, close to the QCP, the proposed scenario adequately explains the low$T$ data on the spin susceptibility, predicting $\chi^{-1}(T) \sim$ $T^{\alpha}$ in the critical density region with a critical exponent $\alpha \simeq 2 / 3$. The spin-fluctuation model (SFM) fails to produce $\alpha<1$. Further, our scenario explains the scaling behavior $\chi^{-1} \sim T^{\alpha} F(H / T)$ of the spin susceptibility in static magnetic fields, whereas the SFM fails to do so. Finally, within the scenario advanced here, FL behavior is recovered close to the QCP by imposing a tiny magnetic field satisfying $\mu_{B} H>T$. In the SFM there is no such provision for reinstating FL theory.

\section{CONCLUSION}

The publication fifteen years ago of the first article $\mathrm{e}^{15}$ on the theory of fermion condensation, in which NFL behavior was deduced from the Landau approach itself, triggered a wave of the criticism and disbelief. The judgment, "This theory is an artifact of the Hartee-Fock method" was typical. By now the debates on this subject have become pointless: numerical solution of the Landau equation (12) is the best way to calm down the critics. Due to lack of space, we present here only a phase diagram of the two-dimensional homogeneous electron gas calculated from Eq. (12). This phase diagram, as plotted in Fig. 4, essentially coincides with predictions of the theory of fermion condensation. Distinctions occur only at extremely low temperatures in the region adjacent to the QCP (for details see Ref. 16). Thus, it is fair to say that the theory of fermion condensation is just another chapter of Fermi-liquid theory. Accordingly, we abandon the conventional attitude of standard FL theory that properties of Fermi liquids are always similar to those of an ideal Fermi gas, since as we have seen, there exists a whole region of densities where this is not the case. Still to be answered is the important question: is the Fermi-liquid approach ${ }^{1}$ relevant to the experimental situation in strongly correlated Fermi systems close to the QCP and beyond it? With the passage of time, a positive answer seems to be more and more evident.

We thank A. Chubukov, P. Coleman, P. Fulde, H. Li, K. Kikoin, G. Lonzarich, J. Thompson, J. Saunders, F. Steglich, and G. Stewart for fruitful discussions. This research was supported by Grant No. NS-8756.2006.2 (VAK and MVZ) from the Russian Ministry of Education and Science and the McDonnell Center for the Space Sciences (VAK).
1 L. D. Landau, JETP 30, 1058, (1956); Sov. Phys. JETP, 3, 920, (1957).

2 D. Pines and P. Nozières, Theory of quantum liquids, v. 1, (W. A. Benjamin, New York-Amsterdam, 1966).

3 C. Bäuerle, Yu. M. Bun'kov, A. S. Chen, S. N. Fisher, and H. Godfrin, J. Low Temp. 110, 333 (1998).

4 A. Casey, H. Patel, J. Nyeki, B. P. Cowan, and J. Saunders, Phys. Rev. Lett. 90, 115301 (2003).

5 O. Prus, Y. Yaish, M. Reznikov, U. Srivan, and V. M. Pudalov, Phys. Rev. B 67, 205407 (2003).

6 J. A. Hertz, Phys. Rev. B 14, 1165 (1976).

7 A. J. Millis, Phys. Rev. B 48, 7183 (1993).
${ }^{8}$ P. Coleman, C. Pepin, Q. Si, and R. Ramazashvili, J. Phys.: Condens. Matter 13, R723 (2001).

9 R. Kü̈hler, N. Geischler, P. Gegenwart, T. Cichorek, K. Neumaier. O. Tegas, C. Geibel, J. A. Mydosh, F. Steglich, L. Zhu, and Q. Si, Phys. Rev. Lett. 91, 066405 (2003).

10 P. Gegenwart, J. Custers, T. Tayama, K. Tenya, C. Geibel, O. Trovarelli, F. Steglich, and K. Neumaier, Acta Phys. Pol. B 34, 323 (2003).

11 D. Takahashi, S. Abe, H. Mizuno, D. A. Tayurski, K. Matsumoto, H. Suzuki, and Y. Onuki, Phys. Rev. B 67, 180407 (2003). 
12 N. J. Curro, T. Caldwell, E. O. Bauer, L. A. Morales, M. J. Graf, Y. Bang, A. V. Balatsky, J. D. Thompson, and J. L. Sarrao, Nature 434, 622 (2005).

13 V. A. Khodel, J. W. Clark, Haochen Li and M. V. Zverev, cond-mat 0609583 .

14 A. B. Migdal, Theory of Finite Fermi Systems and Applications to Atomic Nuclei (Wiley, New York, 1967).

15 V. A. Khodel and V. R. Shaginyan, JETP Letters 51, 553 (1990); Condensed Matter Theories 12, 221 (1997).

16 M. V. Zverev and M. Baldo, JETP 87, 1129 (1998); J. Phys.: Condens. Matter 11, 2059 (1999).

17 V. V. Borisov and M. V. Zverev, JETP Letters, 81, 503 (2005).

18 J. W. Clark, V. A. Khodel, and M. V. Zverev, Phys. Rev. 71, 012401 (2004).

19 V. R. Shaginyan, JETP Letters 77, 99 (2003); 79, 344 (2004).

20 J. Custers, P. Gegenwart, H. Wilhelm, K. Neumaier, Y. Tokiwa, O. Trovarelli, F. Steglich, C. Pepin, and P. Coleman, Nature 424, 524 (2003).

21 G. E. Volovik, JETP Letters 53, 222 (1991).
22 P. Nozières, J. Phys. I France 2, 443 (1992).

23 V. A. Khodel, V. V. Khodel, and V. R. Shaginyan, Phys. Rep. 249, 1 (1994).

${ }^{24}$ M. V. Zverev and V. A. Khodel, JETP Lett. 79, 635 (2004).

25 M. V. Zverev, V. A. Khodel, V. R. Shaginyan and M. Baldo, JETP Lett. 65 (1997) 863.

26 N. Oeschler, P. Gegenwart, M. Lang, R. Movshovich, J. L. Sarrao, J. D. Thompson, and F. Steglich, Phys. Rev. Lett. 91, 076402 (2003).

27 R. Küchler, N. Oeschler, P. Gegenwart, T. Cichorek, K. Neumaier, O. Tegus, C. Geibel, J. A. Mydosh, F. Steglich, L. Zhu, and Q. Si, Phys. Rev. Lett. 91, 066405 (2003); 93, 096402 (2004).

28 V. A. Khodel, M. V. Zverev, and V. M. Yakovenko, Phys. Rev. Lett. 95, 236402 (2005).

29 C. Petrovic, P. G. Pagliuso, M. F. Hundley, R. Movshovich, J. L. Sarrao, J. D. Thompson, Z. Fisk, and P. Monthoux, J. Phys.: Condens. Matter 13, L337 (2001). 